\title{
Uncertain Event Presentation and ECA Modeling in Clinical Pathway Variation

\author{
Runqi Cao
}

School of Economics and Management, Beijing Jiaotong University, Beijing, 100044, China

14120620@bjtu.edu.cn

Keywords: Clinical Pathway; ECA; variation management.

\begin{abstract}
Clinical pathway computer modeling, implementation and control is an important way to enhance clinical pathway implementation effect and also a cardinal channel to achieve efficient medical services. In the process of the research above, find an effective way to manage the variation of clinical pathway is necessary. At present, the variation management mainly based on reasoning methods with predicate logic, its ability to handle uncertain event is lacking. This article discusses how to use fuzzy number to present the uncertain factor in clinical pathway variation event, and embed this presentation in ECA (Event-Condition-Action) model, namely UC-ECA. It's a theoretical model that can control the uncertain factor in reasoning. With this model, we can provide a more optimized reasoning system that can be imported into the already existing clinical pathway system, such as ontology knowledge system.
\end{abstract}

\section{Introduction}

In the early stage, clinical pathway has achieved the effect of the follows, shortening the number of days of hospitalization, saving the cost of medical care, achieving the target of treatment and guaranteeing the quality of nursing. After 20 years of development, clinical pathway has become an effective tool for hospital quality management and disease diagnosis and evaluation standards. So far, more and one hundred China hospitals have used the management model [4].

In the process of clinical pathway modeling. Clinical pathway varies among different individuals, that means when a computer program intent to simulate a pathway in reality, it has to decide what to do when an input dataset is unexpected, fortunately if variation dataset is specific, rules can be made to handle these exceptions [1]. But if the variation is inenarrable, or has many inenarrable factors in it, how could these rules be made? For example, how could we represent 'patient has a poor spirit' in clinical pathway system? There are lots of statements and conditions in actual clinical procedure that cannot be easily qualified or classified. In this article, I call these statements and conditions Uncertain Event. Opposite to a certain event, an uncertain event is that cannot be easily bounded, thus, cannot be easily determined as happened or not happened.

This article gives a solution with fuzzy number theory. By present them as fuzzy number, these ambiguous statement or event in clinical pathway system can be treated as a normal condition, and can participate in predicate operation. I also embed this theory in ECA reasoning model. These already existing acknowledge ontology system, semantic reasoning rules in database, can compatible with this model very well.

\section{Clinical Pathway Variation}

\subsection{Definitions.}

Clinical Pathway, namely Critical Paths, is optimal treatment process taken in the most appropriate time and specific clinical situation by doctors, nurses and other professional. It is a task oriented, structured treatment plan and working mode. Clinical Pathway Variation can be understood as the follows, variation is the deviation between actual treatment process and the standard clinical pathway hypothesis, and it's the changes out of expectations in clinical process [3]. 


\subsection{Problems.}

In the process of computerized clinical pathway management and its system implementation, many scholars have begun to combine the semantic network technology with electronic medical records, in order to build the clinical path knowledge database [7]. In such a knowledge system, according to the medical index system [2], scholar express medical knowledge as an ontology model, and classify variation with different standards. Then they use semantic reasoning technology to simulate the occurrence of variations and also simulate management measures [6].

In practical applications, there are still many problems. On one hand, ambiguous semantic statement must be well handled to support the existing predicate logic, on the second, the variety of clinical pathway variation varies from different aspects, and we should use different means for different types of variation in our model.

\subsection{Methods.}

In this article, an event contains an unclear statement is called uncertain event. I use fuzzy number to present an unclear statement. The concept of fuzzy sets was first proposed by Zadeh, which is an extension of classical set to deal with fuzzy concepts and phenomena quantitatively [8]. And I use ECA (Event-Condition-Action) model as the reasoning engine. Combined with the uncertain event, this model can be called UC-ECA model. UC-ECA reasoning model can provide a more optimized reasoning system that can be imported into the already existing clinical pathway knowledge system.

\section{UC-ECA reasoning model}

\subsection{Classify the variation.}

The goal of this article is to study the variation and processing mechanism based on fuzzy reasoning, and to promote the automatic scheduling and dynamic management of patient treatment process based on clinical pathway knowledge. It is necessary to clarify the range of variation types can be applied to the method.

Variations in the actual clinical pathway are usually quite complex. The causes, properties, or manifestations varies, the differences between manners in which patients are treated, the impact of the treatment process is also different.

As an aspect of evidence-based medicine practice, variations can be classified into two parts roughly, expected-variations and unexpected-variations. The expected variation means that, patient's departure from standardized workflow can be foreseen with the existing practice and related knowledge. Unexpected variation refers to events that have not occurred in previous medical practice, it's often requiring temporary analysis and decision-making by medical personnel, and, most likely cause patient treatment has to be withdrawn from clinical pathways. Unexpected variations usually involve extensive historical information, up-to-date medical knowledge, or similar patient cases that were rare in the past. Hence it is generally assumed that unexpected variation is infrequent.

The expected variation can be classified into four categories according to their source.

- $\quad$ Patient-related variation. Refers to the patients or their families' subjective requirements. Such as do not comply with the requirements of the hospital, refusing to accept specific treatment activities, fasting and so on.

- Staff-related variation. Refers to doctors, nurses and other professionals in the medical decision-making, such as error behaviors, staff or workload changes.

- $\quad$ Source-related variation. Refers to the medical service agencies, such as material resources like equipment, beds and other related activities affecting the implementation of clinics.

- Clinical variations. Refers to the clinical diagnosis and treatment of patients with abnormal conditions, such as patients with low platelet count, wound infection, bleeding, persistent pain and so on.

Expected variations can be deal with reasoning method such as ECA model, and unexpected variations could not. This article shows how UC-ECA model handle uncertain event in expected variations (Figure 1). 


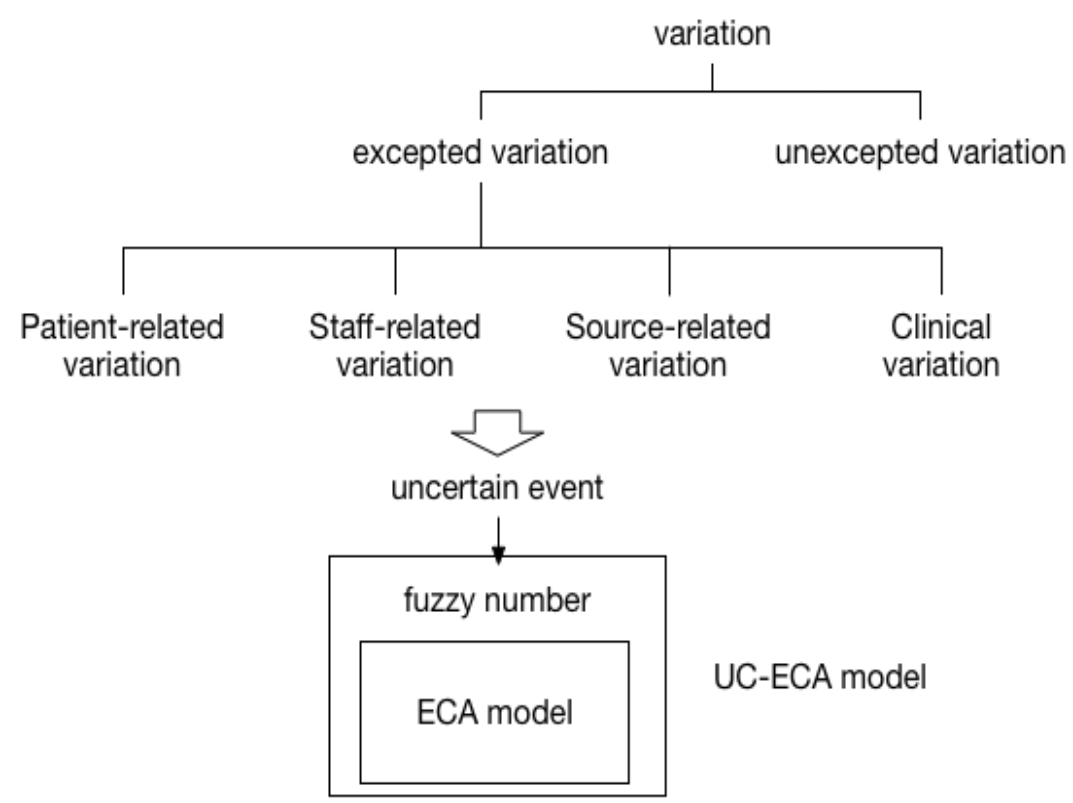

3.2 UC-ECA model.

Fig. 1 variation classification

In this study, the ECA rule model was used to support variation processing. It does not make any assumptions and restrictions on the activities and the standardized clinical path workflow. The change of the clinical pathway system will not influence the implement of UC-ECA model here. So that the UC-ECA based variation process and the clinical pathway workflow implementation are independent of each other. Meanwhile, The UC-ECA rule provides the ability to interact with variation of clinical pathway workflow execution.

In the general form of ECA rules, events and conditions contain clearly defined propositions, traditional or explicit boundary events and conditions. The event occurrence and the result of the condition evaluation are either true or false. However, in clinical pathway variation processing, event and conditional propositions relate to the state of environment such as patient, health care provider, or relate to the implementation of a particular clinical measure. These are usually ambiguous, indeterminate, like abdominal pain, a large amount of bleeding, poor medical condition. These propositions are no longer true, but reflect a certain degree of truth, which can be defined as a real value from zero to one.

This article uses uncertain-ECA model which include fuzzy set method. UC-ECA model can indicates the imprecise knowledge as well as precise knowledge designed in the variation process. A UC-ECA model can be described as follows.

\begin{tabular}{|rl|}
\hline ON & event or uncertain event \\
IF & condition [logical-op (math-op uncertain-condition threshold)] \\
THEN & action
\end{tabular}

In the formula above, a logic-op is a logical operator such as AND, NOT and OR. Square bracket means it's optional. A math-op is a mathematical operator like MAX, MIN and EQUALS.

The event part can make an explicit event described as a classical binary proposition, or a fuzzy event described as a fuzzy proposition. Likewise, the conditional part can make the deterministic condition of the classical proposition, or the ambiguous condition of the fuzzy proposition. When all the events and conditions in a rule are classical propositions, the rules are called general or traditional ECA rules. When they are fuzzy propositions, they are called UC-ECA rules.

Here is an example that a patient applies for blood transfusion. At first it's a normal ECA rule.

\begin{tabular}{|rl|}
\hline ON & apply for blood transfusion \\
IF & Coagulation disorders \\
THEN & do blood transfusion \\
\hline
\end{tabular}

Events, conditions, actions all can be atomic or composite. Atomic events are minimum event instances, while composite events consist of atomic event components. The combination of atomic 
event and composite event is implemented by the classical logical conjunctions. A composite event has rich expression ability. For example, (A AND (NOT B)) OR (NOT A) is consist of two atomic event $\mathrm{A}$ and $\mathrm{B}$. We can expand the UC-ECA rules above, so that it can express more.

ON apply for blood transfusion

IF Coagulation disorders AND surgery soon after

THEN do blood transfusion OR increase the dose

In actual practice, surgical condition is full of uncertainty, the surgery may delay due to the scheduling of operating room most likely. So we can express the condition 'surgery soon after' as an uncertain condition, we use fuzzy number to represent this uncertain condition. Here we predict the time of the soon-coming surgery as a fuzzy number $\widetilde{D}$. The operating room scheduling cycle varies from hospital to hospital, so $\widetilde{\mathrm{D}}$ here is a triangular fuzzy number, which can be expressed as follows.

$$
\mu_{\widetilde{D}}(x)=\left\{\begin{array}{cc}
\frac{x-l}{m-l} & l \leq x<m \\
\frac{u-x}{u-m} & m \leq x \leq u \\
0 & \text { other }
\end{array}\right.
$$

The formulas above means the time of the surgery is $l$ to $u$ days after, and $\operatorname{ker}(\widetilde{D})=m$.

It can be seen from the above discussion that the UC-ECA reasoning model can embed logical operations and semantic uncertainties very well.

\subsection{Calculation on UC-ECA.}

In the UC-ECA model, both the certain condition and the uncertain condition exist, that is, the real number and the fuzzy number exist at the same time. We can use the kernel of fuzzy number as an approximation of the real field.

Given a certain condition $C$, it's kernel is:

$\operatorname{ker}(C)=\{x \mid x \in C\}$

Given a uncertain condition $\widetilde{U C}$, it's kernel is:

$\operatorname{ker}(\widetilde{U C})=\left\{x \mid \mu_{\widetilde{U C}}(x)=1\right\}$

And define the relationship:

$\widetilde{U C}>m \stackrel{\text { def }}{=} \min (\operatorname{ker}(\widetilde{U C}))>m$, and $\widetilde{U C}<m \stackrel{\text { def }}{=} \max (\operatorname{ker}(\widetilde{U C}))<m$

So we can determine a uncertain event true or false by:

$(\widetilde{U C 1}>m)$ and $(\widetilde{U C 2}>m) \stackrel{\text { def }}{=} \min (\operatorname{ker}(\widetilde{U C 1}), \operatorname{ker}(\widetilde{U C 2}))>m$

$(\widetilde{U C 1}<m)$ and $(\widetilde{U C 2}<m) \stackrel{\text { def }}{=} \max (\operatorname{ker}(\widetilde{U C 1}), \operatorname{ker}(\widetilde{U C 2}))<m$

\section{Summary}

In the present study, a specific medical domain ontology can provide a consistent semantic model for the standardization of clinical pathway management and variation management. This article aims to study the uncertain event representation in variation of clinical pathway during computerized implementation, the specific medical domain ontology is not explained in detail. This article makes a good classification of clinical pathway variation and recommend a ECA-like model to handle several types of variation problems. One method does not apply to all types, UC-ECA has certain limitations, and that's the direction of future research.

\section{References}

[1] HQ Wang, JS Li, YF Zhang, et al. Creating personalised clinical pathways by semantic interoperability with electronic health records. Artificial intelligence in medicine. Vol. 58 (2013) No. 2, p. 81-89.

[2] Minghui Liang, Ting Su, Yahui Jiao, et al. American international medical service quality indictor, Chinese Hospitals, Vol. 13(2009) No.4, p. 14-17. 
[3] Lizhen Fang, et al. Clinical Pathway: The New Clinical Service Model. Hunan Science and Technology Press, 2002, p. 13-14.

[4] Information on: http://www.ch-cp.org.cn/m.php?name=hospital\&mo_order=9\&page=1

[5] Fengjie Di. Clinical pathway variation management. Modern Instruments, Vol. 18(2012) No.1, p.20-21.

[6] WeiJuan Fu: Research of Knowledge Reasoning Engine Based on Clinical Diagnosis. Master, Xidian University, China, 2014. P.21-23.

[7] Zhenzhen Feng, Xichuan Zhen, et al. Research on Construction of Intelligent Clinical Pathway Knowledge Database based on Ontology. Chinese Digital Medicine. Vol. 7(2012) No.1, p.78-82.

[8] Zadeh L. A., Fuzzy Sets. Information and Control, Vol. 7(1965), p.338-353. 